\title{
Propagation of Torsional Surface Waves under the Effect of Irregularity and Initial Stress
}

\author{
Shishir Gupta, Dinesh K. Majhi, Sumit K. Vishwakarma, Santimoy Kundu \\ Department of Applied Mathematics, Indian School of Mines, Dhanbad, India \\ E-mail:shishir_ism@yahoo.com \\ Received August 24, 2011; revised October 14, 2011; accepted October 23, 2011
}

\begin{abstract}
The present paper has been framed to study the influence of irregularity, initial stress and porosity on the propagation of torsional surface waves in an initially stressed anisotropic poro-elastic layer over a semi-infinite heterogeneous half space with linearly varying rigidity and density due to irregularity at the interface. The irregularity has been taken in the half-space in the form of a parabola. It is observed that torsional surface waves propagate in this assumed medium. In the absence of irregularity the velocity of torsional surface wave has been obtained. Further, it has been seen that for a layer over a homogeneous half space, the velocity of torsional surface waves coincides with that of Love waves.
\end{abstract}

Keywords: Irregularity, Torsional Surface Waves, Anisotropic, Initial Stress

\section{Introduction}

The study of surface waves in a half-space is important to seismologists due to its possible applications in Geophysical prospecting and in understanding the cause and estimation of damage due to earthquakes. Surface waves carry the greatest amount of energy from shallow shocks and are of primary cause of destruction that can result from earthquakes. The propagation of surface waves in detail are well documented in the text book literature (Achenbach, J. D. [1], Ewing, W. M., Jardetzky, W. S., Press, F. [2], Bath, M. [3]). One type of surface wave may be available in non-homogeneous earth known as torsional surface waves. These waves are horizontally polarized but give a twist to the medium when it propagates. Although much information is available on the propagation of surface waves such as Rayleigh waves, Love waves and Stonely waves etc., the torsional wave has not drawn much attention and very little literature is available on the propagation of this wave. Some papers have been published on the propagation of torsional waves in elastic medium with different types of inhomogeneity. Lord Rayleigh [4] in his remarkable paper showed that the isotropic homogeneous elastic half-space does not allow a torsional surface wave to propagate, in this connection Georgiadis et al. [5] have examined the torsional surface wave in a linear gradient-elastic halfspace. Meissner [6] pointed out that in an inhomogeneous elastic half-space with quadratic variation of shear modulous and density varying linearly with depth, torsional surface waves do exist. Vardoulakis, I. [7] has studied the problem on torsional surface waves in inhomogeneous elastic media. Also S. Dey et al. [8] studied the propagation of torsional waves in a homogeneous substratum over a heterogeneous half-space.

The study of porous medium in recent time has acquired prime interest. The layer of the earth usually of such materials and the medium is generally dealt under the name of poro-elastic medium. Investigation on propagation of waves in liquid saturated porous solids are relevant to geophysical prospecting methods, survey techniques are very useful in oil industry. While deriving the mechanics of such medium it is assumed that the pore sizes are small and macroscopially speaking their average distribution is uniform. The role of pore water in seismology has been emphasized in many studies. Biot [9] has established the theory of the propagation of elastic waves in a porous elastic solid saturated by a viscous fluid. Under the assumption of dynamic coupling between solid and fluid mass, Biot [10] has developed the mathematical theory for the propagation of elastic waves in a fluid saturated porous medium. Based on this theory, many problems of surface waves in poro-elastic materials have been studied in the past years by Buckingham, M. J. [11], Sharma, M. D. and Gogna, M. L. [12], Sharma, M. D., Kumar, R. and Gogna, M. L. [13], Sharma, M. D., Kumar, R. and Gogna, M. L. [14].

The development of initial stresses in the medium is 
due to many reasons, for example resulting from the difference of temperature, process of quenching, shot peening and cold working, slow process of creep, differential external forces, gravity variations etc. These stresses have a pronounced influence on the propagation of waves as shown by Biot [15]. The earth is also an initially stressed medium. It is therefore of much interest to study the influence of these stresses on the propagation of torsional surface wave. The study of surface waves in an initially stressed medium is of interest not for theoretical taste only but for practical purposes too. Based on the pioneering work of Biot [15] on pre-stressed solids, various studies of body and surface wave propagation in the prestressed solids have been carried out by many researchers such as Chattopadhyay et al. [16], Kar, B. K. and Kalyani, V. K. [17] and Dey, S. and Addy, S. K. [18] .

The study of wave propagation in elastic medium with different irregularities is of great importance to seismologists as well as to geophysicists to understand and predict the seismic behavior at the different margins of earth. This fact leads us towards this study. The present paper discusses the possibility of existence of torsional surface wave in an initially stressed anisotropic poro-elastic layer over a semi-infinite heterogeneous half space with linearly varying rigidity and density due to irregularity at the interface. The irregularity has been taken in the halfspace in the form of a parabola. It is observed that torsional surface waves propagate in this assumed medium. Irregularity and initial stress play an important role in the seismic wave propagation. This paper has been framed out to show the effect of irregularity at the interface in the parabolic form and initial stress on the propagation of torsional surface wave. As the porosity parameter decreases, the half-space will become an elastic solid with less pores and the velocity of torsional surface waves decreases and ultimately vanishes when the medium is elastic solid. The effect of irregularity and the initial stresses are very prominent on the propagation of torsional surface waves. The presence of initial compressive stress reduces the velocity of torsional surface waves. As the initial compressive stress increases, the velocity of propagation decreases. It is also observed that in the limiting case if the porous medium changes to a liquid layer then a torsional surface wave don't exist. It is interesting to note that in a poro-elastic medium over a homogeneous half-space the torsional wave mode changes to Love wave mode. Parabolic irregularity is mostly found in the earth so the present study can help the seismologists to understand and predict the seismic behaviour at different margins of the earth.

\section{Formulation of the Problem}

Let us Consider a model which consists of a water satu- rated porous layer $\mathrm{M}_{1}$ of thickness $\mathrm{H}$ with anisotropy of Weiskopf type under compressive initial stresses

$P^{\prime}=-s_{r r}^{\prime}$ along the radial direction and with one parabolic irregularity on the interface between the layer and a semi-infinite non-homogeneous elastic half-space $\mathrm{M}_{2}$ as shown in Figure 1. The heterogeneity has been considered both in density and rigidity. We assume that the irregularity is of the form of a parabola with span of length $2 m$ and depth $h$.

Assuming the origin of the cylindrical co-ordinate system at the middle point of the interface irregularity and the z-axis downward positive, the following variation in rigidity and density has been taken

1) for the layer, $\mu=\mu_{0}, \rho=\rho_{0}$

2) for the half-space, $\mu=\mu_{1}(1+a z), \rho=\rho_{1}(1+b z)$ where $\mu$ and $\rho$ are rigidity and density of the media respectively and $a, b$ are constants having dimensions that are inverse of length.

The equation of irregularity has been taken as

$$
z=\varepsilon F(r) \text {, where } F(r)=\left\{\begin{array}{l}
h\left(1-\frac{r^{2}}{m^{2}}\right) \text { for }|r| \leq m \\
0 \text { for }|r|>m
\end{array}\right.
$$

and $\varepsilon=\frac{h}{2 m}$ and $\varepsilon \ll 1$.

\section{Solution of the Problem}

\subsection{Solution for Porous Layer}

The dynamical equations of initially stressed poroelastic medium is obtained by suitably coupling Biot's [19] dynamical equations of an initially stressed medium with the equations of poro-elasticity given by Weiskopf [20] and Biot $[9,10]$. Those are

$$
\begin{aligned}
& \frac{\partial s_{r r}^{\prime}}{\partial r}+\frac{1}{r} \frac{\partial s_{r \theta}^{\prime}}{\partial \theta}+\frac{\partial s_{r z}^{\prime}}{\partial z}+\frac{s_{r r}^{\prime}-s_{\theta \theta}^{\prime}}{r}-P^{\prime} \frac{\partial \omega_{\theta}^{\prime}}{\partial z} \\
& =\frac{\partial^{2}}{\partial t^{2}}\left(\rho_{r r} u_{r}^{\prime}+\rho_{r \theta} U_{r}^{\prime}\right) \\
& \frac{\partial s_{r \theta}^{\prime}}{\partial r}+\frac{1}{r} \frac{\partial s_{\theta \theta}^{\prime}}{\partial \theta}+\frac{\partial s_{\theta z}^{\prime}}{\partial z}+\frac{2}{r} s_{r \theta}^{\prime}-P^{\prime} \frac{\partial \omega_{z}^{\prime}}{\partial r} \\
& =\frac{\partial^{2}}{\partial t^{2}}\left(\rho_{r r} v_{\theta}^{\prime}+\rho_{r \theta} V_{\theta}^{\prime}\right) \\
& \frac{\partial s_{r z}^{\prime}}{\partial r}+\frac{1}{r} \frac{\partial s_{\theta z}^{\prime}}{\partial \theta}+\frac{\partial s_{z z}^{\prime}}{\partial z}+\frac{1}{r} s_{r z}^{\prime}-P^{\prime} \frac{\partial \omega_{\theta}^{\prime}}{\partial r} \\
& =\frac{\partial^{2}}{\partial t^{2}}\left(\rho_{r r} w_{z}^{\prime}+\rho_{r \theta} W_{z}^{\prime}\right)
\end{aligned}
$$

and

$$
\frac{\partial s^{\prime}}{\partial r}=\frac{\partial^{2}}{\partial t^{2}}\left(\rho_{r \theta} u_{r}^{\prime}+\rho_{\theta \theta} U_{r}^{\prime}\right)
$$




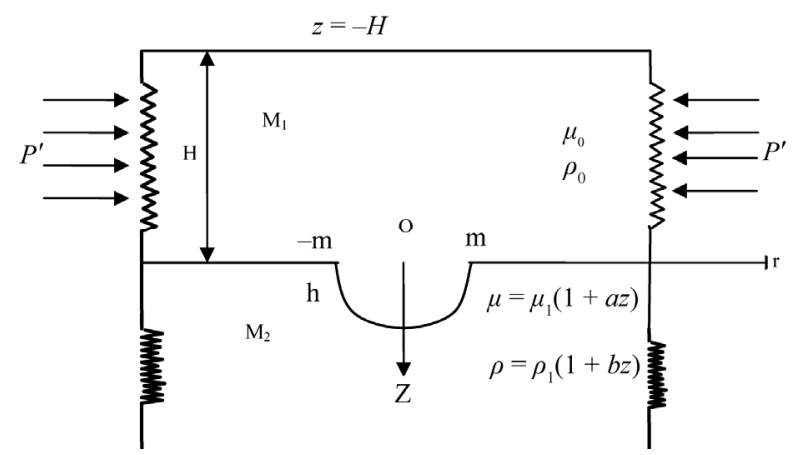

Figure 1. Geometry of the problem.

$$
\begin{aligned}
& \frac{\partial s^{\prime}}{\partial \theta}=\frac{\partial^{2}}{\partial t^{2}}\left(\rho_{r \theta} v_{\theta}^{\prime}+\rho_{\theta \theta} V_{\theta}^{\prime}\right) \\
& \frac{\partial s^{\prime}}{\partial z}=\frac{\partial^{2}}{\partial t^{2}}\left(\rho_{r \theta} w_{z}^{\prime}+\rho_{\theta \theta} W_{z}^{\prime}\right)
\end{aligned}
$$

where $s_{i j}^{\prime}=$ incremental stress components;

$\left(u_{r}^{\prime}, v_{\theta}^{\prime}, w_{z}^{\prime}\right)=$ components of the displacement vector of the solid;

$\left(U_{r}^{\prime}, V_{\theta}^{\prime}, W_{z}^{\prime}\right)=$ components of the displacement vector of the liquid;

$s^{\prime}=$ stress in the liquid,

and

$$
\begin{aligned}
& \omega_{r}^{\prime}=\frac{1}{2 r}\left[\frac{\partial w_{z}^{\prime}}{\partial \theta}-r \frac{\partial v_{\theta}^{\prime}}{\partial z}\right], \omega_{\theta}^{\prime}=\frac{1}{2}\left[\frac{\partial u_{r}^{\prime}}{\partial z}-\frac{\partial w_{z}^{\prime}}{\partial r}\right] \\
& \omega_{z}^{\prime}=\frac{1}{2 r}\left[\frac{\partial\left(r v_{\theta}^{\prime}\right)}{\partial r}-\frac{\partial v_{\theta}^{\prime}}{\partial \theta}\right]
\end{aligned}
$$

are the components of the rotational vector $\omega^{\prime}$.

The stress-strain relations for the water saturated anisotropic porous layer under normal initial stress $P^{\prime}$ are

$$
\begin{aligned}
& s_{r r}^{\prime}=\left(A+P^{\prime}\right) e_{r r}+\left(A-2 N+P^{\prime}\right) e_{\theta \theta}+\left(F+P^{\prime}\right) e_{z z}+Q_{\varepsilon} \\
& s_{\theta \theta}^{\prime}=(A-2 N) e_{r r}+A e_{\theta \theta}+F e_{z z}+Q_{\varepsilon} \\
& s_{z z}^{\prime}=F e_{r r}+F e_{\theta \theta}+C e_{z z}+Q_{\varepsilon} \\
& s_{r \theta}^{\prime}=2 N e_{r \theta}, s_{\theta z}^{\prime}=2 G e_{\theta z}, s_{r z}^{\prime}=2 G e_{z r}
\end{aligned}
$$

where $A, F, C, N$ and $G$ are elastic constants of the medium. $N$ and $G$ are, in particular, shear moduli of the anisotropic layer in the radial and the z-direction respectively.

$$
\begin{aligned}
& e_{r r}=\frac{\partial u_{r}^{\prime}}{\partial r}, e_{\theta \theta}=\frac{1}{r} \frac{\partial v_{\theta}^{\prime}}{\partial \theta}+\frac{u_{r}^{\prime}}{r}, e_{\mathrm{zz}}=\frac{\partial w_{z}^{\prime}}{\partial z} \\
& e_{r \theta}=\frac{1}{2}\left[\frac{1}{r} \frac{\partial u_{r}^{\prime}}{\partial \theta}+\frac{\partial v_{\theta}^{\prime}}{\partial r}-\frac{v_{\theta}^{\prime}}{r}\right], e_{\theta z}=\frac{1}{2}\left[\frac{\partial v_{\theta}^{\prime}}{\partial z}+\frac{1}{r} \frac{\partial w_{z}^{\prime}}{\partial \theta}\right] \\
& e_{z r}=\frac{1}{2}\left[\frac{\partial w_{z}^{\prime}}{\partial r}+\frac{\partial u_{r}^{\prime}}{\partial z}\right]
\end{aligned}
$$

Further, $Q_{\varepsilon}$ being the measure of coupling between the volume change of the solid and the liquid is a positive quantity. $\boldsymbol{s}^{\prime}$ is the stress vector due to the liquid. The stress vector $\boldsymbol{s}^{\prime}$ is related to the fluid pressure $\boldsymbol{P}$ by the relation

$$
-\boldsymbol{s}^{\prime}=f \boldsymbol{P}
$$

where $f$ is porosity of the layer.

The mass coefficients $\rho_{r r}, \rho_{r \theta}$ and $\rho_{\theta \theta}$ are related to the densities $\rho, \rho_{s}, \rho_{w}$ of the layer, the solid and the water respectively by

$$
\rho_{r r}+\rho_{r \theta}=(1-f) \rho_{s}, \rho_{r \theta}+\rho_{\theta \theta}=f \rho_{w}
$$

so that the mass density of the aggregate is

$$
\rho^{\prime}=\rho_{s}+f\left(\rho_{w}-\rho_{s}\right)
$$

The above relation shows that in case the fluid of lighter density $\left(\rho_{w}\right)$ is filled up in the solid matrix of density $\left(\rho_{s}\right)$ then the density of the aggregate $\left(\rho^{\prime}\right)$ will be less than the density of the solid $\left(\rho_{s}\right)$, there may be the case of heavier fluid such as mercury, molten metal etc. filled in the solid matrix, when the density of the aggregate will be more than that of solid. Further this relation shows that as the porosity factor $f$ decreases from 1 to 0 i.e. as the volume of pores decreases, the density of the aggregate tends to the density of the solid. It has been shown by Biot that the mass coefficients obey the following inequalities also

$$
\rho_{r r}>0, \rho_{\theta \theta}>0, \rho_{r \theta}<0, \rho_{r r} \rho_{\theta \theta}-\rho_{r \theta}^{2}>0
$$

For torsional surface waves propagating along the radial direction having displacement of the particles along $\theta$ direction we have,

$$
\left.\begin{array}{l}
u_{r}^{\prime}=0, w_{z}^{\prime}=0, v_{\theta}^{\prime}=v^{\prime}(r, z, t) \\
U_{r}^{\prime}=0, W_{z}^{\prime}=0, V_{\theta}^{\prime}=V^{\prime}(r, z, t)
\end{array}\right\}
$$

The above displacements will produce $e_{\theta z}$ and $e_{r \theta}$ strain components and the other strain components will be zero. Hence the stress-strain relations are

$$
s_{\theta z}^{\prime}=2 G e_{\theta z}, s_{r \theta}^{\prime}=2 N e_{r \theta}
$$

Using (15) in (1) to (6) the equations of motion, which are not automatically satisfied, are

$$
\begin{gathered}
\frac{\partial s_{r \theta}^{\prime}}{\partial r}+\frac{\partial s_{\theta z}^{\prime}}{\partial z}+\frac{2}{r} s_{r \theta}^{\prime}-P^{\prime} \frac{\partial \omega_{z}^{\prime}}{\partial r}=\frac{\partial^{2}}{\partial t^{2}}\left(\rho_{r r} v_{\theta}^{\prime}+\rho_{r \theta} V_{\theta}^{\prime}\right) \\
\frac{\partial^{2}}{\partial t^{2}}\left(\rho_{r \theta} v_{\theta}^{\prime}+\rho_{\theta \theta} V_{\theta}^{\prime}\right)=0
\end{gathered}
$$

Using stress-strain relations (8), (16) may be written as

$$
\begin{aligned}
& \left(N-\frac{P^{\prime}}{2}\right)\left(\frac{\partial^{2} v_{\theta}^{\prime}}{\partial r^{2}}-\frac{v_{\theta}^{\prime}}{r^{2}}+\frac{1}{r} \frac{\partial v_{\theta}^{\prime}}{\partial r}\right)+G \frac{\partial^{2} v_{\theta}^{\prime}}{\partial z^{2}} \\
& =\frac{\partial^{2}}{\partial t^{2}}\left(\rho_{r r} v_{\theta}^{\prime}+\rho_{r \theta} V_{\theta}^{\prime}\right)
\end{aligned}
$$


From (17), we have

$$
\begin{gathered}
\rho_{r \theta} v_{\theta}^{\prime}+\rho_{\theta \theta} V_{\theta}^{\prime}=d^{\prime \prime}(\text { say }) \\
\therefore V_{\theta}^{\prime}=\frac{d^{\prime \prime}-\rho_{r \theta} v_{\theta}^{\prime}}{\rho_{\theta \theta}}
\end{gathered}
$$

Now,

$$
\frac{\partial^{2}}{\partial t^{2}}\left(\rho_{r r} v_{\theta}^{\prime}+\rho_{r \theta} V_{\theta}^{\prime}\right)=d^{\prime} \frac{\partial^{2}\left(v_{\theta}^{\prime}\right)}{\partial t^{2}},
$$

where $d^{\prime}=\rho_{r r}-\frac{\rho_{r \theta}^{2}}{\rho_{\theta \theta}}$

Using (19) in (18), we get

$$
\left(N-\frac{P^{\prime}}{2}\right)\left(\frac{\partial^{2} v_{\theta}^{\prime}}{\partial r^{2}}-\frac{v_{\theta}^{\prime}}{r^{2}}+\frac{1}{r} \frac{\partial v_{\theta}^{\prime}}{\partial r}\right)+G \frac{\partial^{2} v_{\theta}^{\prime}}{\partial z^{2}}=d^{\prime} \frac{\partial^{2} v_{\theta}^{\prime}}{\partial t^{2}}
$$

From Equation (20) it is clear that the velocity of the shear wave along radial(r) direction is $\left[\frac{N-\frac{P^{\prime}}{2}}{d^{\prime}}\right]^{\frac{1}{2}}$ and that along z-direction is $\left(\frac{G}{d^{\prime}}\right)^{\frac{1}{2}}$. Now the shear wave velocity $\beta^{\prime}$ in the porous medium along radial direction may be expressed as,

$\beta^{\prime}=\left[\frac{1-\zeta}{d} c_{\beta}^{2}\right]^{\frac{1}{2}}$, where $c_{\beta}=\sqrt{\frac{N}{\rho^{\prime}}}$ is the velocity of the shear wave in the corresponding initial stress free non-porous anisotropic elastic medium along the radial direction, $\zeta=\frac{P^{\prime}}{2 N}$ is the non-dimensional parameter due to the initial stress $P^{\prime}$ and the density ratio,

$$
d=\frac{d^{\prime}}{\rho^{\prime}}=\frac{1}{\rho^{\prime}}\left(\rho_{r r}-\frac{\rho_{r \theta}^{2}}{\rho_{\theta \theta}}\right)=\gamma_{11}-\frac{\gamma_{12}^{2}}{\gamma_{22}}
$$

where $\gamma_{11}=\frac{\rho_{r r}}{\rho^{\prime}}, \gamma_{12}=\frac{\rho_{r \theta}}{\rho^{\prime}}, \gamma_{22}=\frac{\rho_{\theta \theta}}{\rho^{\prime}}$ are the non-dimensional parameters for the material of the porous layer as obtained by Biot.

Introducing the non-dimensional radial and depth coordinates we have

$$
R=\frac{r}{L}, \xi=\xi_{0}+\frac{Z}{L},
$$

$\xi_{0}$ being a constant and $\frac{1}{L}$ being wave number where $k=\frac{1}{L}$, the Equation (20) may be written as

$$
\frac{\partial^{2} v_{\theta}^{\prime}}{\partial R^{2}}+\frac{1}{R} \frac{\partial v_{\theta}^{\prime}}{\partial R}-\frac{v_{\theta}^{\prime}}{R^{2}}+\frac{G}{N-\frac{P^{\prime}}{2}} \frac{\partial^{2} v_{\theta}^{\prime}}{\partial \xi^{2}}=\frac{d^{\prime} L^{2}}{N-\frac{P^{\prime}}{2}} \frac{\partial^{2} v_{\theta}^{\prime}}{\partial t^{2}}
$$

We assume a solution of Equation (21) of the form $v_{\theta}^{\prime}=v_{2}^{\prime}(\xi) J_{1}(R) \mathrm{e}^{i \omega t}$, where $J_{1}(R)$ is the Bessel function of the first kind and $v_{2}^{\prime}(\xi)$ is the solution of the following equation

$$
\frac{\mathrm{d}^{2} v_{2}^{\prime}}{\mathrm{d} \xi^{2}}+q^{2} v_{2}^{\prime}=0
$$

where $q^{2}=\frac{\left(\frac{\omega^{2} L^{2} d^{\prime}}{N-\frac{P^{\prime}}{2}}-1\right)}{\frac{G}{N-\frac{P^{\prime}}{2}}}$

The solution of Equation (22) may be taken as

$$
v_{2}^{\prime}(\xi)=A e^{i q \xi}+B e^{-i q \xi}
$$

Therefore, for the torsional surface wave propagating in the radial direction, the solution of Equation (21) may be taken as

$$
v_{\theta}^{\prime}=J_{1}(R)\left(A e^{i q \xi}+B e^{-i q \xi}\right) e^{i \omega t}
$$

where $q^{2}=d v\left(\frac{c^{2}}{c_{\beta}^{2}}-\frac{1-\zeta}{d}\right)$, where $v=\frac{N}{G}, c=\omega L$ is the phase velocity of the torsional surface wave.

\subsection{Solution for Non-Homogeneous Elastic Half Space}

The lower medium is considered as non-homogeneous elastic half space. The Equaiton of motion may be written as

$$
\frac{\partial \sigma_{r \theta}}{\partial r}+\frac{2}{r} \sigma_{r \theta}+\frac{\partial \sigma_{z \theta}}{\partial z}=\rho \frac{\partial^{2} v}{\partial t^{2}}
$$

where $\sigma_{r \theta}$ and $\sigma_{z \theta}$ are stress components in the half space, $v(r, \theta, z)$ is the displacement and $\rho$ is the density of the material of the half space.

The non-homogeneity in the medium are taken as

$$
\mu=\mu_{1}(1+a z), \rho=\rho_{1}(1+b z)
$$

where, $\mu_{1}$ and $\rho_{1}$ are the values of $\mu$ and $\rho$ respectively at $z=0$, and $a, b$ are constants having dimensions that are inverse of length.

Using the stress-strain relations

$$
\sigma_{r \theta}=\mu\left[\frac{\partial v}{\partial r}-\frac{v}{r}\right], \sigma_{z \theta}=\mu \frac{\partial v}{\partial z}
$$


and the relations (25), the Equation of motion (24) may be written as

$$
\frac{\partial^{2} v}{\partial r^{2}}+\frac{1}{r} \frac{\partial v}{\partial r}-\frac{v}{r^{2}}+\frac{\partial^{2} v}{\partial z^{2}}+\frac{a}{1+a z} \frac{\partial v}{\partial z}=\frac{\rho_{1}(1+b z)}{\mu_{1}(1+a z)} \frac{\partial^{2} v}{\partial t^{2}}
$$

Introducing the non-dimensional co-ordinates

$$
R=\frac{r}{L} \text { and } \xi=\xi_{0}+\frac{Z}{L},
$$

Equation (27) takes the form as

$$
\begin{aligned}
& \frac{\partial^{2} v}{\partial R^{2}}+\frac{1}{R} \frac{\partial v}{\partial R}-\frac{v}{R^{2}}+\frac{\partial^{2} v}{\partial \xi^{2}}+\frac{L a}{\left[1+a\left(\xi-\xi_{0}\right) L\right]} \frac{\partial v}{\partial \xi} \\
& =\frac{\rho_{1} L^{2}}{\mu_{1}} \frac{1+b\left(\xi-\xi_{0}\right) L}{1+a\left(\xi-\xi_{0}\right) L} \frac{\partial^{2} v}{\partial t^{2}}
\end{aligned}
$$

We assume a solution of Equation (28) of the form

$$
v=v_{2}(\xi) J_{1}(R) e^{i \omega t},
$$

where $v_{2}$ is the solution of the following equation

$$
\begin{aligned}
& \frac{\mathrm{d}^{2} v_{2}}{\mathrm{~d} \xi^{2}}+\frac{a L}{1+a\left(\xi-\xi_{0}\right) L} \frac{\mathrm{d} v_{2}}{\mathrm{~d} \xi} \\
& +\left[\frac{c^{2}}{c_{1}^{2}} \frac{1+b\left(\xi-\xi_{0}\right) L}{1+a\left(\xi-\xi_{0}\right) L}-1\right] v_{2}=0
\end{aligned}
$$

In the above, $c=\omega L$ is the phase velocity of the torsional surface wave and $c_{1}=\sqrt{\frac{\mu_{1}}{\rho_{1}}}$, velocity of shear wave in an initially stress-free elastic medium and $J_{1}(R)$ is the Bessel function of the first kind.

$$
\text { Substituting } v_{2}(\xi)=\frac{L^{-\frac{1}{2}} \phi(\xi)}{\left[\frac{1}{L}+a\left(\xi-\xi_{0}\right)\right]^{\frac{1}{2}}} \text { in the Equa- }
$$

tion (29) to eliminate the term $\frac{\mathrm{d} v_{2}}{\mathrm{~d} \xi}$,

we obtain

$$
\phi^{\prime \prime}(\xi)+\left[\frac{a^{2}}{4\left\{\frac{1}{L}+a\left(\xi-\xi_{0}\right)\right\}^{2}}+\frac{c^{2}}{c_{1}^{2}} \frac{\frac{1}{L}+b\left(\xi-\xi_{0}\right)}{\frac{1}{L}+a\left(\xi-\xi_{0}\right)}-1\right] \phi(\xi)
$$$$
=0
$$

Using $\phi(\xi)=\psi(\eta)$ in (30), where

$\eta=\frac{2 p}{a}\left[\frac{1}{L}+a\left(\xi-\xi_{0}\right)\right]$ we get

$$
\psi^{\prime \prime}(\eta)+\left[\frac{1}{4 \eta^{2}}+\frac{s}{\eta}-\frac{1}{4}\right] \psi(\eta)=0
$$

where $s=\frac{c^{2}(a-b) L}{2 c_{1}^{2} p a^{2} L^{2}}, p^{2}=1-\frac{b}{a} \frac{c^{2}}{c_{1}^{2}}$

Equation (31) is Whittaker's equation.

We are interested in the solution of Equation (31) which is bounded and vanishes as $z \rightarrow \infty$, therefore we search for the solution which gives $v(z) \rightarrow 0$ as $z \rightarrow \infty$. This condition is equivalent to $\lim _{\eta \rightarrow \infty} \psi(\eta) \rightarrow 0$.

Therefore the solution of Equation (31) satisfying the above condition may be written as

$$
v=\frac{D_{1} W_{s, 0}(\eta)}{L^{\frac{1}{2}}\left(\frac{a \eta}{2 p}\right)^{\frac{1}{2}}} J_{1}(R) e^{i \omega t}
$$

\section{Boundary Conditions and Dispersion Equation}

The boundary conditions are as follows

$$
\begin{aligned}
& \text { 1) } \mu_{0}\left(\frac{\partial v_{\theta}^{\prime}}{\partial z}\right)=0 \text { at } z=-H \\
& \text { 2) } v_{\theta}^{\prime}=v \text { at } z=\varepsilon F(r) \\
& \text { 3) }\left(\sigma_{r \theta}\right)_{0}=\left(\sigma_{r \theta}\right)_{1} \text { at } z=\varepsilon F(r)
\end{aligned}
$$

where

$$
\begin{gathered}
\left(\sigma_{r \theta}\right)_{0}=l \mu_{0} \frac{\partial v_{\theta}^{\prime}}{\partial \theta}+n \mu_{0} \frac{\partial v_{\theta}^{\prime}}{\partial \mathrm{z}}, \\
\left(\sigma_{r \theta}\right)_{1}=l \mu_{1} \frac{\partial v}{\partial \theta}+n \mu_{1} \frac{\partial v}{\partial \mathrm{z}}
\end{gathered}
$$

and $(l, 0, n)$ are components of unit normal (to the interface at $z=0$ ).

$$
\begin{gathered}
l=0, n=1 \text { at } z=-H \\
l=-\frac{\varepsilon F^{\prime}}{\sqrt{1+\varepsilon^{2} F^{\prime 2}}}, n=\frac{1}{\sqrt{1+\varepsilon^{2} F^{\prime 2}}} ; \\
\text { at } z=\varepsilon F(r)
\end{gathered}
$$

where $F^{\prime}=\frac{\mathrm{d} F}{\mathrm{~d} r}$

The boundary condition (3) may be written as

$$
\begin{aligned}
& \mu_{0}\left(\frac{\partial v_{\theta}^{\prime}}{\partial r}-\frac{v_{\theta}^{\prime}}{r}\right)\left(-\frac{\varepsilon F^{\prime}}{\sqrt{1+\varepsilon^{2} F^{\prime 2}}}\right)+\frac{1}{\sqrt{1+\varepsilon^{2} F^{\prime 2}}} \mu_{0} \frac{\partial v_{\theta}^{\prime}}{\partial z} \\
& =\mu_{1}\left(\frac{\partial v}{\partial r}-\frac{v}{r}\right)\left(-\frac{\varepsilon F^{\prime}}{\sqrt{1+\varepsilon^{2} F^{\prime 2}}}\right)+\frac{1}{\sqrt{1+\varepsilon^{2} F^{\prime 2}}} \mu_{1} \frac{\partial v}{\partial z}
\end{aligned}
$$

at $z=\varepsilon F(r)$

Now, using boundary conditions (1), (2) and (3) respectively we get 


$$
\begin{gathered}
A e^{i q\left(\xi_{0}-\frac{H}{L}\right)}-B e^{-i q\left(\xi_{0}-\frac{H}{L}\right)}=0 \\
A e^{i q \xi}+B e^{-i q \xi}=\frac{D_{1} W_{s, 0}(\eta)}{(1+a \varepsilon F(r))^{\frac{1}{2}}} \\
A M_{1} \mathrm{e}^{i q \xi}-B N_{1} \mathrm{e}^{-i q \xi}=D_{1} K_{1}
\end{gathered}
$$

where

$$
\begin{aligned}
K_{1}=\frac{\mu_{1}}{\mu_{0}}\left[\frac{W_{s, 0}(\eta)}{(1+a \varepsilon F(r))^{1 / 2}}\left(\frac{2 r h \varepsilon}{m^{2}}\right)\left\{\frac{1}{L} J_{1}^{\prime}\left(\frac{r}{L}\right)-\frac{1}{r} J_{1}(R)\right\}\right. \\
+\left(\frac{2 p}{a L}\right)^{s} J_{1}(R) e^{-p / a L(1+a \varepsilon F(r))}(1+a z)^{s-\frac{1}{2}} \\
\quad\left\{\frac{a^{2} L}{8 p} \frac{(s-0.5)^{2}(s+0.5)(3.5-s)}{(1+a \varepsilon F(r))^{2}}\right. \\
\quad-\frac{a^{3} L^{2}}{8 p^{2}} \frac{(s-0.5)^{2}(s-1.5)^{2}(2.5-s)}{(1+a \varepsilon F(r))^{3}} \\
+\frac{a(s-0.5)(s+1.5)}{2(1+a \varepsilon F(r))}-\frac{p}{L} \\
\left.\left.\quad-\frac{a^{2} L}{2 p} \frac{(s-0.5)^{3}}{(1+a \varepsilon F(r))^{2}}\right\}\right]
\end{aligned}
$$

Now, eliminating $A, B$ and $D_{1}$ from Equations (33), (34) and (35) we get

$$
\left|\begin{array}{llr}
e^{i q\left(\xi_{0}-\frac{H}{L}\right)} & -e^{-i q\left(\xi_{0}-\frac{H}{L}\right)} & 0 \\
e^{i q \xi} & e^{-i q \xi} & -\frac{W_{\mathrm{s}, 0}(\eta)}{(1+a \varepsilon F(r))^{\frac{1}{2}}} \\
M_{1} e^{i q \xi} & N_{1}-e^{-i q \xi} & -K_{1}
\end{array}\right|=0
$$

Expanding the Whittaker function $W_{s, 0}(\eta)$ up to linear terms in $\eta$, Equation (37) reduces to

$$
\operatorname{tank} q(\varepsilon F(r)+\mathrm{H})=\frac{1}{q}\left[R_{1}-\frac{\mu_{1}}{\mu_{0}} \frac{R_{2}}{R_{3}}\right]
$$

where

$$
\begin{gathered}
R_{1}=\frac{-2 r h \varepsilon}{m^{2}}\left(1-\frac{\mu_{1}}{\mu_{0}}\right)\left(\frac{R}{4}+\frac{R^{3}}{96}+\frac{R^{5}}{1536}\right), \\
R_{2}=\left(\frac{a^{2} L^{2}}{8 p} \frac{(s-0.5)^{2}(s+0.5)(3.5-s)}{(1+a \varepsilon F(r))^{2}}\right. \\
-\frac{a^{3} L^{3}}{8 p^{2}} \frac{(s-0.5)^{2}(s-1.5)^{2}(2.5-s)}{(1+a \varepsilon F(r))^{3}}, \\
\left.+\frac{a L(s-0.5)(s+1.5)}{2(1+a \varepsilon F(r))}-p-\frac{a^{2} L^{2}}{2 p} \frac{(s-0.5)^{3}}{(1+a \varepsilon F(r))^{2}}\right) \\
R_{3}=\left(1-\frac{a L}{2 p} \frac{(s-0.5)^{2}}{(1+\varepsilon F(r))}+\frac{a^{2} L^{2}}{8 p^{2}} \frac{(s-0.5)^{2}(s-1.5)^{2}}{(1+\varepsilon F(r))^{2}}\right) .
\end{gathered}
$$

\section{Particular Cases}

If the medium is non-porous then $f \rightarrow 0$ and $\rho_{s} \rightarrow \rho^{\prime}$ which leads to $\gamma_{11}+\gamma_{12} \rightarrow 1$ and $\gamma_{11}+\gamma_{12} \rightarrow 0$ and hence $\left(\gamma_{11}-\frac{\gamma_{12}^{2}}{\gamma_{22}}\right) \rightarrow 1$ giving $d \rightarrow 1$.

Again if $f \rightarrow 1$ then $\rho_{w} \rightarrow \rho^{\prime}$ and the medium becomes fluid. In this case, the velocity of shear wave in the medium can not exist when $d=\left(\gamma_{11}-\frac{\gamma_{12}^{2}}{\gamma_{22}}\right) \rightarrow 0$.

Therefore, for a non-porous solid $d=1$, for fluid $d=0$ and for porous solid filled with liquid $0<d<1$.

\subsection{Case I}

If $\varepsilon \rightarrow 0, h \rightarrow 0$ i.e. in the absence of irregularity in the half space, then (38) reduces to

$$
\tan (k q H)=\frac{\mu_{1} p}{\mu_{0} q} \frac{\left[1-\frac{a L}{2 p}(s-0.5)(s+1.5)+\frac{a^{2} L^{2}}{8 p^{2}}(s-0.5)^{2}(s+2.5)(s-1.5)-\frac{a^{3} L^{3}}{8 p^{3}}(s-0.5)^{2}(s-1.5)^{2}(s-2.5)\right]}{1-\frac{a L}{2 p}(s-0.5)^{2}+\frac{a^{2} L^{2}}{8 p^{2}}(s-0.5)^{2}(s-1.5)^{2}}
$$

which is the same velocity equation in initially stressed anisotropic heterogeneous poroelastic medium as obtained by Dey \& Sarkar (2002).

\subsection{Case II}

If $\varepsilon \rightarrow 0, a \rightarrow 0, b \rightarrow 0$ i.e. when half space is free from irregularity and also half space is homogeneous then from (38) we get

$$
\tan (k q H)=\frac{\mu_{1} p}{\mu_{0} q},
$$

where $p^{2}=1-\frac{c^{2}}{c_{1}^{2}}, q^{2}=d v\left(\frac{c^{2}}{c_{\beta}^{2}}-\frac{1-\zeta}{d}\right)$ 
which is the dispersion equation of Love wave in an initially stressed poro-elastic medium.

\subsection{Case III}

If $P^{\prime} \neq 0, a \rightarrow 0, b \rightarrow 0$ i.e. in the presence of normal initial stress and when the half-space is homogeneous then Equation (38) reduces to

$$
\begin{aligned}
& \tan [k q(\varepsilon F(r)+H)]=\frac{\mu_{1} p}{\mu_{0} q}, \\
& \text { where } p^{2}=1-\frac{c^{2}}{c_{1}^{2}} \text { and } q^{2}=d v\left(\frac{c^{2}}{c_{\beta}^{2}}-\frac{1-\zeta}{d}\right)
\end{aligned}
$$

which is the velocity equation of Love wave in this case.

\subsection{Case IV}

If $P^{\prime}=0, a \rightarrow 0, b \rightarrow 0$ i.e. in the absence of normal initial stress and when the half-space is homogeneous then from (38) we get

$$
\begin{aligned}
& \tan [k q(\varepsilon F(r)+H)]=\frac{\mu_{1} p}{\mu_{0} q}, \\
& \text { where } p^{2}=1-\frac{c^{2}}{c_{1}^{2}} \text { and } q^{2}=d v\left(\frac{c^{2}}{c_{\beta}^{2}}-\frac{1}{d}\right)
\end{aligned}
$$

\subsection{Case V}

If $\varepsilon \rightarrow 0, a \rightarrow 0, b \rightarrow 0, \zeta \rightarrow 0, d \rightarrow 1$ and $N=G$ i.e. when the half-space is homogeneous and free from irregularity and also when the layer is initial stress free, isotropic and non-porous then (38) reduces to

$$
\tan (k q H)=\frac{\mu_{1} p}{\mu_{0} q},
$$

where

$$
\begin{gathered}
p^{2}=1-\frac{c^{2}}{c_{1}^{2}}, q^{2}=\frac{c^{2}}{c_{\beta}^{2}}-1 \\
\text { or, } \tan \left[\left(\frac{c^{2}}{c_{\beta}^{2}}-1\right)^{\frac{1}{2}} k H\right]=\frac{\mu_{1}}{\mu_{0}} \frac{\left(1-\frac{c^{2}}{c_{1}^{2}}\right)^{\frac{1}{2}}}{\left(\frac{c^{2}}{c_{\beta}^{2}}-1\right)^{\frac{1}{2}}}
\end{gathered}
$$

which is the well-known classical result of Love wave.

\section{Numerical Computation and Discussion}

To study the consolidated effect of porosity, irregularity and initial stresses on the propagation of the torsional surface waves, the numerical computation of velocity $c^{2} / c_{\beta}^{2}$ has been made from (38) under different values of $d, a L, b L, N / G, h / H, \quad \mu_{1} / \mu_{0}, r / m, h / m, k m$, $\varepsilon, \zeta$ and $c_{\beta}^{2} / c_{1}^{2}=0.5$. The results are presented in Figures 2 to 5 .

Figure 2 gives the dispersion curves for increasing values of $\mathrm{kH}$ at different sizes of the irregularity and compares the results in the medium with and without initial stresses. The curve numbers 1,2 and 3 are for an initially stress-free medium, and the curve numbers 4,5 and 6 are for the initially stressed medium with $\zeta=0.4$. The figure shows that the effect of the irregularity and the initial stresses are very prominent on the propagation of torsional surface waves. The presence of initial com-

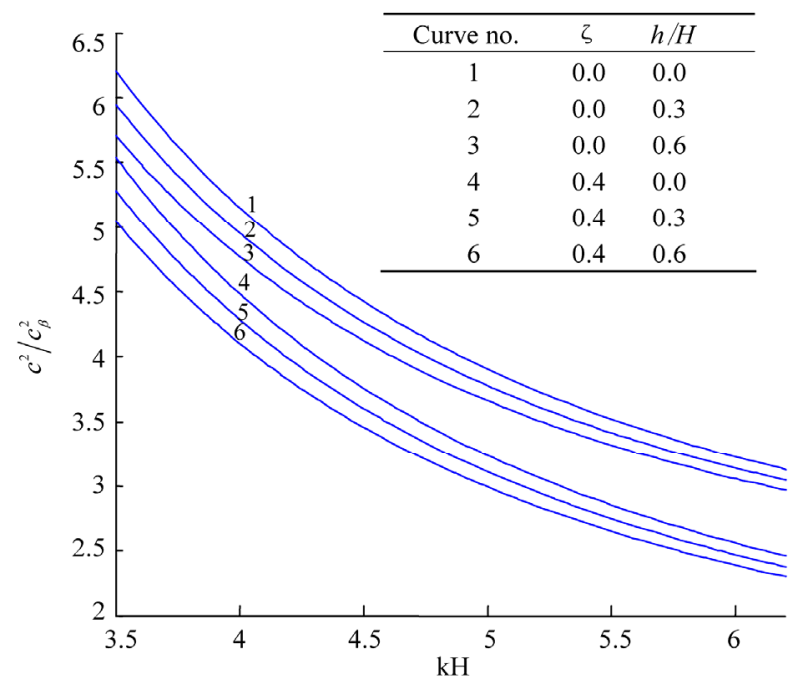

Figure 2. Torsional wave dispersion curves at different initial stresses and at different sizes of irregularity $\mu_{1} / \mu_{0}=0.4$, $N / G=0.3, d=0.6, a / k=0.1, b / k=0.1, \varepsilon=0.1, r / m=0.02$, $h / m=0.03, k m=0.01$.

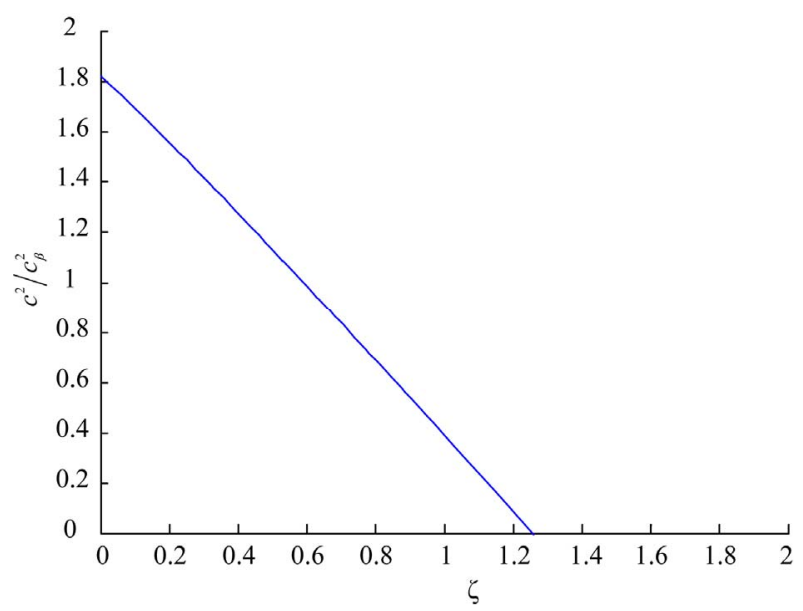

Figure 3. Variation of velocity of torsional wave with respect to initial compressive stress for $k H=0.72, N / G=2, d$ $=0.6, a L=0.1, b L=0.1, h / H=0.3, \varepsilon=0.1, r / m=0.01, h / m=$ $0.02, k m=0.01, \mu_{1} / \mu_{0}=0.4$. 


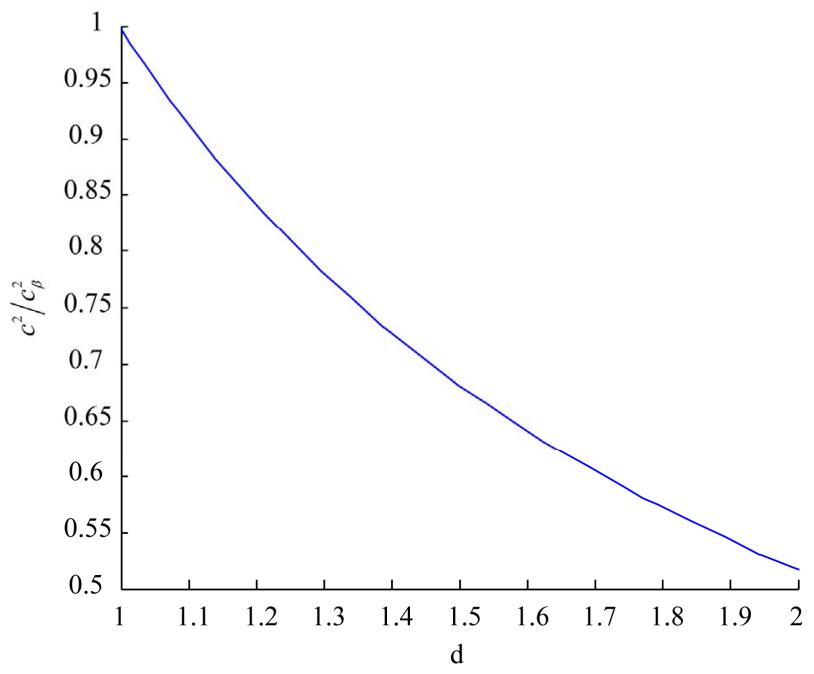

Figure 4. Variation of velocity of torsional wave with respect to porosity for $k H=0.72, N / G=2, \zeta=0.6, a L=0.1, b L$ $=0.1, h / H=0.3, \varepsilon=0.1, r / m=0.01, h / m=0.02, k m=0.01$, $\mu_{1} / \mu_{0}=0.4$.

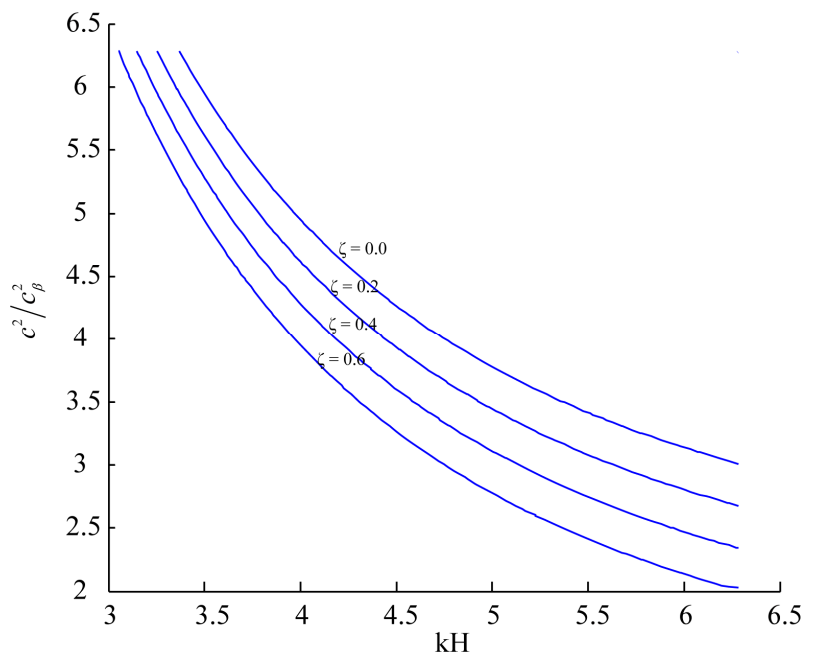

Figure 5. Effect of initial stress on the velocity of torsional wave for $\mu_{1} / \mu_{0}=0.4, N / G=0.3, d=0.6, a / k=0.1, b / k=0.1, \varepsilon$ $=0.1, r / m=0.02, h / m=0.03, k m=0.01, h / H=0.3$.

pressive stress reduces the velocity of torsional surface waves. The presence of the liquid-filled pore in the medium also reduces the velocity.

Figure 3 gives a variation of velocity of torsional surface waves for the variation of $\zeta$. The curve confirm that as the initial stress parameter increases, the velocity decreases.

Figure 4 gives a variation of velocity of torsional surface waves for the variation of porosity. The curve confirm that as the porosity increases, the velocity decreases.

Figure 5 gives the dispersion curves at different values of $k H$. This shows that as $k H$ increases, the velocity drops down. Hence it is expected that after certain thickness of the layer, the torsional surface wave will not propagate in the medium. This figure also confirms that as the initial compressive stress increases, the velocity of propagation decreases.

\section{Acknowledgements}

The authors convey their sincere thanks to Indian School of Mines, Dhanbad, for providing JRF, Mr. Dinesh Kumar Majhi and also facilitating us with best facility.

\section{References}

[1] J. D. Achenbach, "Wave Propagation in Elastic Solids," North Holland Publishing Comp., New York, 1973

[2] W. M. Ewing, W. S. Jardetzky and F. Press, "Elastic Waves in LAYERED Media,” McgrawHill, New York, 1957.

[3] M. Bath, "Mathematical Aspects of Seismology,” Elsvier Publishing Comp., New York, 1968.

[4] L. Rayleigh, "On Waves Propagated along Plane Surface of an Elastic Solid,” Proceedings of the London Mathematical Society, Vol. 17, No. 3, 1885, pp. 4-11. doi:10.1112/plms/s1-17.1.4

[5] H. G. Georgiadis, I. Vardoulakis and G. Lykotrafitis "Torsional Surface Waves in a Gradient-Elastic Half Space,” Wave Motion, Vol. 31, No. 4, 2000, pp. 333-348. doi:10.1016/S0165-2125(99)00035-9

[6] E. Meissner, "Elastic Oberflachenwellen Mit Dispersion in Einem Inhomogeneous Medium,” Viertlagahrsschriftder Naturforschenden Gesellschaft, Zurich, Vol. 66, 1921, pp. 181-195.

[7] I. Vardoulakis, “Torsional Surface Waves in Inhomogeneous Elastic Media,” International Journal for Numerical and Analytical Methods in Geomechanics, Vol. 8, No. 3, 1984, pp. 287-296.

[8] S. Dey, A. K. Gupta and S. Gupta, "Propagation of Torsional Surface Waves in a Homogeneous Substratum over a Heterogeneous Half-Space,” International Journal for Numerical and Analytical Methods in Geomechanics, Vol. 20, 1996, pp. 287-294.

doi:10.1002/(SICI)1096-9853(199604)20:4<287::AID-N AG822>3.0.CO;2-2

[9] M. A. Biot, "Theory of Deformation of a Porous Viscoelastic Anisotropic Solid,” Journal of Applied Physics, Vol. 27, No. 5, 1956, pp. 459-467. doi:10.1063/1.1722402

[10] M. A. Biot, "Theory of Propagation of Elastic Waves in a Fluid-Saturated Porous Solid,” Journal of the Acoustical Society of America, Vol. 28, No. 2, 1956, pp. 168-178. doi:10.1121/1.1908239

[11] M. J. Buckingham, "Theory of Compressional and Shear Waves in Fluid-Like Marine Sediments,” Journal of the Acoustical Society of America, Vol. 103, No. 1, 1998, pp. 288-299. doi:10.1121/1.421091

[12] M. D. Sharma and M. L. Gogna, "Propagation of Love Waves in an Initially-Stressed Medium Consisting of 
sandy Layer Lying over a Liquid Saturated Porous Solid Half-Space,” Journal of the Acoustical Society of America, Vol. 89, No. 6, 1991, pp. 2584-2588. doi:10.1121/1.400697

[13] M. D. Sharma, R. Kumar and M. L. Gogna, "Surface Wave Propagation in a Transversely Isotropic Elastic Layer Overlying a Liquid-Saturated Porous Solid HalfSpace and Lying under a Uniform Layer of Liquid," Pure and Applied Geophysics, Vol. 133, No. 3, 1990, pp. 523539. doi:10.1007/BF00878003

[14] M. D. Sharma, R. Kumar and M. L. Gogna, "Surface Wave Propagation in a Liquid-Saturated Porous Layer Over-Lying a Homogeneous Transversely Isotropic HalfSpace and Lying under a Uniform Layer of Liquid,” International Journal of Solids and Structures, Vol. 27, 1991, pp. 1255-1267. doi:10.1016/0020-7683(91)90161-8

[15] M. A. Biot, "Influence of Initial Stress on Elastic Waves,” Journal of Applied Physics, Vol. 11, No. 8, 1940, pp. 522-530. doi:10.1063/1.1712807

\section{Appendix}

$H$ : Thickness of the layer

$\mu$ : Rigidity of the medium

$\rho:$ Density of the medium

$a, b$ : Constants having dimensions that are inverse of length

$s_{i j}^{\prime}$ : Incremental stress components

$e_{i j}$ : Components of strains

$u_{r}^{\prime}, v_{\theta}^{\prime}, w_{z}^{\prime}$ : Displacement components of the solid in the radial, circumferential and axial directions respectively. $U_{r}^{\prime}, V_{\theta}^{\prime}, W_{z}^{\prime}$ : Displacement components of the liquid in the radial, circumferential and axial directions respectively.
[16] A. Chattopadhyay, S. Bose and M. Chakraborty, "Reflection of Elastic Waves under Initial Stress at a Free Surface," Journal of the Acoustical Society of America, Vol. 72, No. 1, 1982, pp. 255-263. doi:10.1121/1.387987

[17] B. K. Kar and V. K. Kalyani, "Reflection and Refraction of SH-Waves Due to the Presence of a Sandwiched Initially Stressed Sandy Layer," Geophysical research bulletin, Vol. 25, No. 3, 1987, pp. 117-124.

[18] S. Dey and S. K. Addy, "Reflection of Plane Waves under Initial Stress at a Free Surface,” International Journal of Non-Linear Mechanics, Vol. 12, No. 6, 1977, pp. 371381. doi:10.1016/0020-7462(77)90038-5

[19] M. A. Biot, "Mechanics of Incremental Deformation," Wiley, New York, 1965.

[20] W. H. Weiskopf, "Stresses in Soils under a Foundation," Journal of the Franklin Institute, Vol. 239, No. 6, 1945, pp. 445-465. doi:10.1016/0016-0032(45)90189-X

$s^{\prime}:$ Stress in the liquid

$\omega_{r}^{\prime}, \omega_{\theta}^{\prime}, \omega_{z}^{\prime}$ : Components of the rotational vector $\omega^{\prime}$ in radial, circumferential and axial directions respectively. $\omega$ : Circular frequency

$k$ : Wave number

$f:$ Porosity of the layer

$c$ : Phase velocity of the torsional surface wave

$c_{1}$ : Velocity of shear wave in an initially stress-free elastic medium.

$R$ : Dimensionless quantity.

$A, B$ and $D_{1}$ : Arbitrary constant 